\title{
On Embeddings of Modular Curves in Projective Spaces
}

\author{
Goran Muić
}




\title{
ON EMBEDDINGS OF MODULAR CURVES IN PROJECTIVE SPACES
}

\author{
GORAN MUIĆ
}

ABstract. We use explicit results on modular forms [8] via uniformization theory to obtain embeddings of modular curves in projective spaces.

\section{INTRODUCTION}

Let $X$ be the upper half-plane. Then the group $S L_{2}(\mathbb{R})$ acts on $X$ as follows:

$$
g . z=\frac{a z+b}{c z+d}, \quad g=\left(\begin{array}{ll}
a & b \\
c & d
\end{array}\right) \in S L_{2}(\mathbb{R}) .
$$

We let $\mu(g, z)=c z+d$. Next, the $S L_{2}(\mathbb{R})$-invariant measure on $X$ is defined by $d x d y / y^{2}$, where the coordinates on $X$ are written in a usual way $z=x+\sqrt{-1} y, y>0$. A discrete subgroup $\Gamma \subset S L_{2}(\mathbb{R})$ is called a Fuchsian group of the first kind if its fundamental domain $\mathcal{F}_{\Gamma}$ in $X$ has a finite volume. Then, adding a finite number of points in $\mathbb{R} \cup\{\infty\}$ called cusps, $\mathcal{F}_{\Gamma}$ can be compactified. In this way we obtain a compact Riemann surface $\mathfrak{R}_{\Gamma}$.

Let $m \geq 3$ be an integer. We consider the space $S_{m}(\Gamma)$ of all modular forms of weight $m$ which are cuspidal i.e., this is a space of all holomorphic functions $f: X \rightarrow \mathbb{C}$ such that $f(\gamma . z)=\mu(\gamma, z)^{m} f(z)(z \in X, \gamma \in \Gamma)$ which are holomorphic and vanish at every cusp for $\Gamma$. The space $S_{m}(\Gamma)$ is a finite-dimensional Hilbert space under the Petersson inner product:

$$
\left\langle f_{1}, f_{2}\right\rangle=\int_{\Gamma \backslash X} y^{m} f_{1}(z) \overline{f_{2}(z)} \frac{d x d y}{y^{2}} .
$$

Let $\xi \in X$ be a fixed point. Then we define the cuspidal modular forms $\Delta_{k, m, \xi}$ in the following way:

$$
\left\langle f, \Delta_{k, m, \xi, \chi}\right\rangle=\left.\frac{d^{k} f}{d z^{k}}\right|_{z=\xi}, \quad f \in S_{m}(\Gamma), \quad k \geq 0 .
$$

In [8] we give the following explicit construction of the modular forms $\Delta_{k, m, \xi}$ :

$$
\Delta_{k, m, \xi}(z)=\epsilon_{\Gamma}^{-1} 2^{m-2} \pi^{-1}(\sqrt{-1})^{m} \prod_{i=0}^{k}(m-1+i) \sum_{\gamma \in \Gamma} \frac{1}{(\gamma . z-\bar{\xi})^{k+m}} \mu(\gamma, z)^{-m},
$$

where $\epsilon_{\Gamma}=\#(\{ \pm 1\} \cap \Gamma)$.

As it is explained in ([8], Section 2), above construction is related to the work of Petersson and refines the construction of Petersson ([13], [14]). We give two rather different proofs of above expansion for $\Delta_{k, m, \xi}$. The one given ([8], Section 2) uses ideas of Selberg, and the one given in the context of representation theory is given in [9] using the methods developed

2000 Mathematics Subject Classification. 11F11, 14H55, 30F35.

Key words and phrases. modular forms, Poincaré series, Riemann surfaces, linear systems. 
in [6]. It is applied in [10] to obtain rather precise estimates on classical Poincaré series which together with a non-vanishing criterion [7] was applied to study non-vanishing of $L-$ functions attached to modular forms. In this paper we give another application of modular forms constructed in [8].

By means of the uniformization theory, we may realize every smooth complex complete curve of genus $g \geq 2$ in the form $\mathfrak{R}_{\Gamma}$, where $\Gamma$ has neither elliptic points nor cusps. Then, a well-known result about the Riemann surfaces says that one can use holomorphic differentials of degree $m \geq 3$ to construct the embeddings of $\mathfrak{R}_{\Gamma}$ into projective spaces. Instead of working with holomorphic differentials of degree $m$, we may work with the space of cuspidal modular forms $S_{2 m}(\Gamma)$ (the isomorphism between the two can be extracted from pages 51, 52 in [4], and it is also well-known). In fact, the idea of using modular forms (via Poincaré series) to construct holomorphic maps on curves is very old one [3]. The advantage working with $S_{m}(\Gamma)$ is that we can allow $\Gamma$ which might have elliptic points and cusps which is the case with usual congruence subgroups $\Gamma_{0}(N)$, and of course we do not have to limit ourselves to the case $m$ is even. So, let $\Gamma$ be any Fuchsian group of the first kind. We let $t_{m}=\operatorname{dim} S_{m}(\Gamma)$. The dimension $t_{m}$ is explicitly computed in ([4], Theorems 2.5.2, 2.5.3) (see Lemma 2-2 (v) in this paper).

Let $m \geq 3$ such that $t_{m} \geq 2$. Then selecting a basis $f_{0}, \ldots, f_{t_{m}-1}$ of $S_{m}(\Gamma)$ one may construct a holomorphic map $\mathfrak{R}_{\Gamma} \longrightarrow \mathbb{P}^{t_{m}-1}$ given by $z \mapsto\left(f_{0}(z): \cdots: f_{t_{m}-1}(z)\right)$. In Section 3 in Theorem 3-3, we prove that for $t_{m} \geq g+1$, where $g$ is the genus of $\mathfrak{R}_{\Gamma}$, this map is associated to the complete linear system $\left|\mathfrak{c}_{f}\right|$. Here, $f$ is an arbitrary non-zero modular form in $S_{m}(\Gamma)$, and $\mathfrak{c}_{f}$ is an (integral) divisor $\mathfrak{c}_{f}$ of degree $t_{m}+g-1$ (see Lemma 2-2 (vi)). Essentially, $\mathfrak{c}_{f}$ is the divisor of zeroes of $f$ when we subtract necessary contribution of elliptic points and cusps common to all non-zero modular forms. The idea of considering such divisors goes back to Petersson [14]. In Section 3 we give some other information related to obtained projective curves: we compute their degrees, field of rational functions and show that the divisors $\mathfrak{c}_{f}$ are nothing else than hyperplane intersection divisors. Section 3 is a preliminary section of the paper and the results are based on the standard theory of algebraic curves [5], and as some results obtained in ([8], Section 4) which are refined and generalized in Section 2.

Section 4 is the central section of the paper. In this section we use above mentioned modular forms $\Delta_{k, m, \xi}$ to construct explicit embeddings into projective spaces

$$
\mathfrak{R}_{\Gamma} \hookrightarrow \mathbb{P}^{k}
$$

where $k$ ranges between $g+1$ and $t_{m}-1$. When $k=t_{m}-1$ we obtain the explicit version of Theorem 3-3. In fact, since our modular forms depend on the parameter $\xi \in X$, we do this a little more generally. Our families of embeddings are $C^{\infty}$ in $\xi$ when $\xi$ ranges over the complement of the set of all $\left(t_{m}-1\right)$-order Weierstrass points [14]. The distribution of such points was studied in [12], [11]. It resembles the distribution of zeroes of eigenforms for $\Gamma=S L_{2}(\mathbb{Z})[16],[2]$. In Section 4, we determine $\left(t_{m}-1\right)$-order Weierstrass points in terms of our modular forms $\Delta_{k, m, \xi}$. 
In Section 5 (see Proposition 5-1) we study the complete linear system attached to above embedding for $k=t_{m}-1$ as a function on $\xi$ on the complement of the set of all $\left(t_{m}-1\right)-$ order Weierstrass points. We use the methods analogous to that in the usual Weierstrass preparation theorem.

\section{Preliminaries}

In this section we recall and refine some results from [8]. We start by recalling some results from $([4], 2.3)$.

Throughout this paper, we write

$$
t_{m}=\operatorname{dim} S_{m}(\Gamma) .
$$

Let $m \geq 2$ be an even integer and $f \in S_{m}(\Gamma)-\{0\}$. Then $\nu_{z-\xi}(f)$ the order of the holomorphic function $f$ at $\xi$. For each $\gamma \in \Gamma$, the functional equation $f(\gamma . z)=\mu(\gamma, z)^{m} f(z)$ shows that $\nu_{z-\xi}(f)=\nu_{z-\xi^{\prime}}(f)$, where $\xi^{\prime}=\gamma \cdot \xi$. Also, if we let

$$
e_{\xi}=\#\left(\Gamma_{\xi} / \Gamma \cap\{ \pm 1\}\right) \text {, }
$$

then $e_{\xi}=e_{\xi^{\prime}}$. The point $\xi$ is elliptic if $e_{\xi}>1$. Next, following ([4], 2.3), we define

$$
\nu_{\xi}(f)=\nu_{z-\xi}(f) / e_{\xi} \text {. }
$$

Clearly, $\nu_{\xi}=\nu_{\xi^{\prime}}$, and we may let

$$
\nu_{\mathfrak{a}_{\xi}}(f)=\nu_{\xi}(f)
$$

where

$$
\mathfrak{a}_{\xi} \in \mathfrak{R}_{\Gamma} \text { is a projection of } \xi \text { to } \mathfrak{R}_{\Gamma},
$$

a notation we use throughout this paper.

If $x \in \mathbb{R} \cup\{\infty\}$ is a cups for $\Gamma$, then we define $\nu_{x}(f)$ as follows. Let $\sigma \in S L_{2}(\mathbb{R})$ such that $\sigma . x=\infty$. We write

$$
\{ \pm 1\} \sigma \Gamma_{x} \sigma^{-1}=\{ \pm 1\}\left\{\left(\begin{array}{cc}
1 & l h^{\prime} \\
0 & 1
\end{array}\right) ; \quad l \in \mathbb{Z}\right\}
$$

where $h^{\prime}>0$. Then we write the Fourier expansion of $f$ at $x$ as follows:

$$
\left(\left.f\right|_{m} \sigma^{-1}\right)(\sigma . z)=\sum_{n=1}^{\infty} a_{n} e^{2 \pi \sqrt{-1} n \sigma . z / h^{\prime}} .
$$

(We remind the reader that $m$ is even.) We let

$$
\nu_{x}(f)=N \geq 0,
$$

where $N$ is defined by $a_{0}=a_{1}=\cdots=a_{N-1}=0, a_{N} \neq 0$. One easily see that this definition does not depend on $\sigma$. Also, if $x^{\prime}=\gamma \cdot x$, then $\nu_{x}(f)=\nu_{x}(f)$. Hence, if $\mathfrak{b}_{x} \in \mathfrak{R}_{\Gamma}$ is a cusp corresponding to $x$, then we may define

$$
\nu_{\mathfrak{b}_{x}}=\nu_{x}(f) .
$$

Put

$$
\operatorname{div}(f)=\sum_{\mathfrak{a} \in \mathfrak{R}_{\Gamma}} \nu_{\mathfrak{a}}(f) \mathfrak{a} \in \mathbb{Q} \otimes \operatorname{Div}\left(\Re_{\Gamma}\right),
$$

where $\operatorname{Div}\left(\mathfrak{R}_{\Gamma}\right)$ is the group of (integral) divisors on $\mathfrak{R}_{\Gamma}$. 
Using ([4], 2.3), this sum is finite i.e., $\nu_{\mathfrak{a}}(f) \neq 0$ for only a finitely many points. We let

$$
\operatorname{deg}(\operatorname{div}(f))=\sum_{\mathfrak{a} \in \mathfrak{R}_{\Gamma}} \nu_{\mathfrak{a}}(f)
$$

Assume now that $-1 \notin \Gamma$ and $m \geq 1$ is odd. Let $f \in S_{m}(\Gamma), f \neq 0$. Then $f^{2} \in S_{2 m}(\Gamma)$. We define $\nu_{\mathfrak{a}}(f)=\nu_{\mathfrak{a}}\left(f^{2}\right) / 2$, and $\operatorname{define} \operatorname{div}(f)$ and $\operatorname{deg}(\operatorname{div}(f))$ as before. (See [4], page 52.)

Let $\mathfrak{d}_{i} \in \mathbb{Q} \otimes \operatorname{Div}\left(\mathfrak{R}_{\Gamma}\right), i=1,2$. Then we say that $\mathfrak{d}_{1} \geq \mathfrak{d}_{2}$ if their difference $\mathfrak{d}_{1}-\mathfrak{d}_{2}$ belongs to $\operatorname{Div}\left(\mathfrak{R}_{\Gamma}\right)$ and is non-negative in the usual sense.

Lemma 2-2. Assume that $m \geq 1$ is an integer and $-1 \notin \Gamma$ if $m$ is odd. Assume that $f \in S_{m}(\Gamma), f \neq 0$. Let $t$ be the number of inequivalent cusps and $u$ (resp., $v$ ) the number of inequivalent regular (resp., irregular) cusps for $\Gamma$. Then we have the following:

(i) For $\mathfrak{a} \in \mathfrak{R}_{\Gamma}$, we have $\nu_{\mathfrak{a}}(f) \geq 0$.

(ii) Let $\mathfrak{a} \in \mathfrak{R}_{\Gamma}$ is a cusp. If $m$ is even or $\mathfrak{a}$ is regular, then $\nu_{\mathfrak{a}}(f) \geq 1$ is an integer. If $\mathfrak{a}$ is not regular, then $\nu_{\mathfrak{a}}(f) \geq 1 / 2$ is in $1 / 2+\mathbb{Z}$.

(iii) If $\mathfrak{a} \in \mathfrak{R}_{\Gamma}$ is not an elliptic point or a cusp, then $\nu_{\mathfrak{a}}(f) \geq 0$ is an integer. If $\mathfrak{a} \in \mathfrak{R}_{\Gamma}$ is an elliptic point, then $\nu_{\mathfrak{a}}(f)-\frac{m}{2}\left(1-1 / e_{\mathfrak{a}}\right)$ is an integer.

(iv) Let $g$ be the genus of $\mathfrak{R}_{\Gamma}$. Then

$$
\operatorname{deg}(\operatorname{div}(f))=m(g-1)+\frac{m}{2}\left(t+\sum_{\mathfrak{a} \in \mathfrak{R}_{\Gamma}, \quad \text { elliptic }}\left(1-1 / e_{\mathfrak{a}}\right)\right) .
$$

(v) Let $[x]$ denote the largest integer $\leq x$ for $x \in \mathbb{R}$. Then,

$$
t_{m}=\left\{\begin{array}{l}
(m-1)(g-1)+\left(\frac{m}{2}-1\right) t+\sum_{\substack{\mathfrak{a} \in \mathfrak{R}_{\Gamma}, \\
\text { elliptic }}}\left[\frac{m}{2}\left(1-1 / e_{\mathfrak{a}}\right)\right], \quad m>2, \text { even } \\
g, m=2,
\end{array}\right.
$$

and

$$
\begin{aligned}
t_{m}= & (m-1)(g-1)+\left(\frac{m}{2}-1\right) u+\left(\frac{m}{2}-\frac{1}{2}\right) v+ \\
& +\sum_{\substack{\mathfrak{a} \in \mathfrak{R}_{\Gamma}, \\
\text { elliptic }}}\left[\frac{m}{2}\left(1-1 / e_{\mathfrak{a}}\right)\right], \quad m \geq 3, \text { odd } .
\end{aligned}
$$

(vi) There exists an integral divisor $\mathfrak{c}_{f} \geq 0$ of degree $t_{m}+g-1$ such that

$$
\begin{aligned}
\operatorname{div}(f)= & \mathfrak{c}_{f}+\sum_{\mathfrak{a} \in \mathfrak{R}_{\Gamma}, \quad \text { elliptic }}\left(\frac{m}{2}\left(1-1 / e_{\mathfrak{a}}\right)-\left[\frac{m}{2}\left(1-1 / e_{\mathfrak{a}}\right)\right]\right) \mathfrak{a}+ \\
& +\left\{\begin{array}{l}
\sum_{\mathfrak{b} \in \mathfrak{R}_{\Gamma}, \mathfrak{b}}, m \geq 2 \text { is even } \\
\sum_{\substack{\text { cusp } \\
\text { regular cusp }}} \mathfrak{b}+\sum_{\substack{\mathfrak{b} \in \mathfrak{R}_{\Gamma}, \\
\text { irregular cusp }}} \frac{1}{2} \mathfrak{b}, \quad m \geq 1 \text { is odd }
\end{array}\right.
\end{aligned}
$$

Proof. This is ([8], Lemma 4-1). 
Definition 2-3. Let $\xi \in X$ or let $\xi$ be a cusp for $\Gamma$. Let $m \geq 1$ be an integer such that $S_{m}(\Gamma) \neq 0$. We define (see Lemma 2-2 (iv))

$$
k_{\xi, m} \stackrel{\text { def }}{=} \sup _{\substack{f \in S_{m}(\Gamma) \\ f \neq 0}} \mathfrak{c}_{f}\left(\mathfrak{a}_{\xi}\right) .
$$

Let $\xi \in X$ be a non-elliptic point. Then $k_{\xi, m}=\sup _{\substack{f \in S_{m}(\Gamma) \\ f \neq 0}} \nu_{z-\xi}(f)$ which agrees with the definition given in ([8], Lemma 4-2). Going back to the general set-up of Definition 2-3, we observe that there exists a unique to a scalar $f_{\xi, m} \in S_{m}(\Gamma)-\{0\}$ such that $c_{f_{\xi, m}}\left(\mathfrak{a}_{\xi}\right)=k_{\xi, m}$. (See [8], Lemma 4-2 for the case $\xi$ non-elliptic; the general case has similar proof.) By Lemma 2-2 (vi), we may write

$$
\begin{aligned}
& \operatorname{div}\left(f_{\xi, m}\right)=\mathfrak{d}_{\xi, m}+k_{\xi, m} \mathfrak{a}_{\xi}+\sum_{\mathfrak{a} \in \mathfrak{R}_{\Gamma}, \text { elliptic }}\left(\frac{m}{2}\left(1-1 / e_{\mathfrak{a}}\right)-\left[\frac{m}{2}\left(1-1 / e_{\mathfrak{a}}\right)\right]\right) \mathfrak{a}+ \\
& +\left\{\begin{array}{l}
\sum_{\substack{\mathfrak{b} \in \mathfrak{R}_{\Gamma}, \\
\text { cusp }}} \mathfrak{b} \quad m \geq 2 \text { is even } \\
\sum_{\substack{\mathfrak{b} \in \mathfrak{R}_{\Gamma}, \\
\text { regular cusp }}}^{\mathfrak{b}} \mathfrak{b}+\sum_{\substack{\mathfrak{b} \in \mathfrak{R}_{\Gamma}, \\
\text { irregular cusp }}} \frac{1}{2} \mathfrak{b}, \quad m \geq 1 \text { is odd, }
\end{array}\right.
\end{aligned}
$$

where $\mathfrak{d}_{\xi, m}$ is a non-negative integral divisor of degree (see Lemma 2-2 (vi))

$$
\operatorname{deg}\left(\mathfrak{d}_{\xi, m}\right)=g-1+t_{m}-k_{\xi, m} \leq g, \text { for } m \geq 3 .
$$

We may define the usual spaces of meromorphic functions

$$
L\left(k \mathfrak{a}_{\xi}+\mathfrak{d}_{\xi, m}\right)=\left\{F \in \mathbb{C}\left(\mathfrak{R}_{\Gamma}\right) ; \quad \operatorname{div}(F)+k \mathfrak{a}_{\xi}+\mathfrak{d}_{\xi, m} \geq 0\right\}, \quad k=0,1,2, \ldots,
$$

where $\mathbb{C}\left(\mathfrak{R}_{\Gamma}\right)$ is the field of rational functions on $\mathfrak{R}_{\Gamma}$.

We have the inclusions

$$
L\left(0 \cdot \mathfrak{a}_{\xi}+\mathfrak{d}_{\xi, m}\right) \subset L\left(1 \cdot \mathfrak{a}_{\xi}+\mathfrak{d}_{\xi, m}\right) \subset \cdots ;
$$

the dimension of the spaces increase for not more than one. We collect some results that has the proofs similar to those given by ([8], Section 4).

Lemma 2-6. Let $\xi \in X$ or let $\xi$ be a cusp for $\Gamma$. Let $m \geq 3$ be an integer such that $S_{m}(\Gamma) \neq 0$. Then

$$
t_{m}-1 \leq k_{\xi, m} \leq t_{m}+g-1 .
$$

Proof. This is ([8], Lemma 4-4) when $\xi$ is not elliptic. In other two cases the upper bound has the same proof. For the lower bound, we need to adjust the proof of ([8], Lemma 4-2(iv)). Instead of working in $S_{m}(\Gamma)$, we work in the space of meromorphic functions $\left\{f / f_{\xi, m} ; f \in S_{m}(\Gamma)\right\}$, where $f_{\xi, m}$ is fixed but the proof is essentially the same. In more details, we restrict to a neighborhood of $\mathfrak{a}_{\xi}$ and observe that the functions from that space of functions have poles from order 0 up to at most $k_{\xi, m}$, choosing a local chart we can switch to the vector space of functions holomorphic at $\mathfrak{a}_{\xi}$ having a zero of order at most $k_{\xi, m}$. Then, one easily adjust the proof ([8], Lemma 4-2(iv)).

We include the following lemma which just the standard Riemann-Roch: 
Lemma 2-7. Let $\xi \in X$ or let $\xi$ be a cusp for $\Gamma$. Let $m \geq 3$ be an integer such that $S_{m}(\Gamma) \neq 0$. Then, for $k \geq g-t_{m}+k_{\xi, m}$, we have the following ${ }^{1}$ :

$$
\operatorname{dim} L\left(k \mathfrak{a}_{\xi}+\mathfrak{d}_{\xi, m}\right)=t_{m}+k-k_{\xi, m} .
$$

Proof. The Riemann-Roch theorem says

$$
\operatorname{dim} L\left(k \mathfrak{a}_{\xi}+\mathfrak{d}_{\xi, m}\right)=\operatorname{deg}\left(k \mathfrak{a}_{\xi}+\mathfrak{d}_{\xi, m}\right)+\operatorname{dim} L\left(K-k \mathfrak{a}_{\xi}-\mathfrak{d}_{\xi, m}\right)+1-g .
$$

Here $K$ is the canonical divisor. The claim is obvious if $g=0$. Indeed, Lemma 2-6 implies that

$$
g-t_{m}+k_{\xi, m}=1 \text {. }
$$

Since, also $K=0$, the Riemann-Roch theorem and (2-5) implies that

$$
\operatorname{dim} L\left(k \mathfrak{a}_{\xi}+\mathfrak{d}_{\xi, m}\right)=t_{m}+k-k_{\xi, m} .
$$

So, we assume that $g \geq 1$. Then, $K \neq 0$ and we have

$$
\operatorname{deg}(K)=2 g-2 .
$$

Thus, as usual, we see that if

$$
\operatorname{deg}\left(K-k \mathfrak{a}_{\xi}-\mathfrak{d}_{\xi, m}\right)=2 g-2-k-\operatorname{deg}\left(\mathfrak{d}_{\xi, m}\right)<0
$$

i.e.,

$$
k \geq 2 g-1-\operatorname{deg}\left(\mathfrak{d}_{\xi, m}\right)=g-t_{m}+k_{\xi, m},
$$

then $\operatorname{dim} L\left(K-k \mathfrak{a}_{\xi}-\mathfrak{d}_{\xi, m}\right)=0$ and with the aid of (2-5) the lemma follows.

Lemma 2-8. Let $\xi \in X$ or let $\xi$ be a cusp for $\Gamma$. Let $m \geq 3$ be an integer such that $S_{m}(\Gamma) \neq 0$. Then, for all $0 \leq i \leq k_{\xi, m}$, we have

$$
L\left(\left(k_{\xi, m}-i\right) \mathfrak{a}_{\xi}+\mathfrak{d}_{\xi, m}\right)=\left\{f / f_{\xi, m} ; \quad f=0 \text { or } \mathfrak{c}_{f}\left(\mathfrak{a}_{\xi}\right) \geq i\right\} .
$$

Moreover, $\operatorname{dim} L\left(\mathfrak{d}_{\xi, m}\right)=1$ i.e., it consists of constants.

Proof. This is proved in the same way as ([8], Lemma 4-13).

Lemma 2-9. Let $\xi \in X$ or let $\xi$ be a cusp for $\Gamma$. Let $m \geq 3$ be an integer such that $S_{m}(\Gamma) \neq 0$. Then, for all $0 \leq i \leq t_{m}-g-1$, there exists $f \in S_{m}(\Gamma)$ such that $\mathfrak{c}_{f}\left(\mathfrak{a}_{\xi}\right)=i$. In particular, if $t_{m} \geq g+1$, then there exists $f \in S_{m}(\Gamma)$ such that $\mathfrak{c}_{f}\left(\mathfrak{a}_{\xi}\right)=0$.

Proof. Indeed, by Lemma 2-7, we have

$$
L\left(\left(k_{\xi, m}-i\right) \mathfrak{a}_{\xi}+\mathfrak{d}_{\xi, m}\right) \neq L\left(\left(k_{\xi, m}-i-1\right) \mathfrak{a}_{\xi}+\mathfrak{d}_{\xi, m}\right)
$$

if $k_{\xi, m}-i-1 \geq g-t_{m}+k_{\xi, m}$. Now, we apply Lemma 2-8.

The following proposition is the main result of the present section. We use notion of linear systems of divisors. We refer to ([5], page 147) for this notion.

\footnotetext{
${ }^{1}$ Lemma $2-2(\mathrm{v})$ and the assumption $S_{m}(\Gamma) \neq 0$ imply that $t_{m} \geq g$. Hence, we can take $k=k_{\xi, m}$.
} 
Proposition 2-10. Let $m \geq 3$ be an integer such that $S_{m}(\Gamma) \neq 0$. Then, for any non-zero $f \in S_{m}(\Gamma)$, we have the following:

$$
L\left(\mathfrak{c}_{f}\right)=\left\{g / f ; \quad g \in S_{m}(\Gamma)\right\} .
$$

Furthermore, if $t_{m} \geq g+1$ the complete linear system $\left|\mathfrak{c}_{f}\right|$ has no base points.

Proof. By definition, we have the following:

$$
L\left(\mathfrak{c}_{f}\right)=\left\{F \in \mathbb{C}\left(\mathfrak{R}_{\Gamma}\right) ; \operatorname{div}(F)+\mathfrak{c}_{f} \geq 0 .\right\}
$$

Next, if $g \in S(\Gamma), g \neq 0$, then by Lemma 2-2 (vi) we obtain

$$
\operatorname{div}\left(\frac{g}{f}\right)+\mathfrak{c}_{f}=\operatorname{div}(g)-\operatorname{div}(f)+\mathfrak{c}_{f}=\mathfrak{c}_{g}-\mathfrak{c}_{f}+\mathfrak{c}_{f}=\mathfrak{c}_{g} \geq 0 .
$$

This shows that $g / f \in L\left(\mathfrak{c}_{f}\right)$.

Let $\xi \in X$ or let $\xi$ be a cusp for $\Gamma$. By Lemma 2-8, we have the following:

$$
\operatorname{dim} L\left(k_{\xi, m} \mathfrak{a}_{\xi}+\mathfrak{d}_{\xi, m}\right)=t_{m}
$$

Again, by Lemma 2-2 (vi), we have

$$
\operatorname{div}\left(f_{\xi, m} / f\right)=k_{\xi, m} \mathfrak{a}_{\xi}+\mathfrak{d}_{\xi, m}-\mathfrak{c}_{f} .
$$

Thus, the divisors $\mathfrak{c}_{f}$ and $k_{\xi, m} \mathfrak{a}_{\xi}+\mathfrak{d}_{\xi, m}$ are linearly equivalent. This implies that

$$
\operatorname{dim} L\left(\mathfrak{c}_{f}\right)=\operatorname{dim} L\left(k_{\xi, m} \mathfrak{a}_{\xi}+\mathfrak{d}_{\xi, m}\right)=t_{m} .
$$

This proves the first claim of the proposition.

The assumption $t_{m} \geq g+1$ implies that for every $\zeta \in X$ or a cusp, there exists $g \in$ $S_{m}(\Gamma)$ such that $\mathfrak{c}_{g}\left(\mathfrak{a}_{\zeta}\right)=0$ (see Lemma 2-9). This means that $\mathfrak{a}_{\zeta} \notin \operatorname{supp}\left(\mathfrak{c}_{g}\right)$ for some $g \in S_{m}(\Gamma)-\{0\}$. Then, by $(2-11)$, we have that $g / f \notin L\left(\mathfrak{c}_{f}-\mathfrak{a}_{\zeta}\right)$. This proves the last claim of the proposition.

\section{Maps to Projective Spaces}

Assume that $m \geq 3$ and $t_{m} \geq 2$. We have a holomorphic map

$$
\mathfrak{R}_{\Gamma} \longrightarrow \mathbb{P}^{t_{m}-1}
$$

defined by

$$
\mathfrak{a}_{z} \mapsto\left(f_{0}(z): \cdots: f_{t_{m}-1}(z)\right)
$$

where $f_{0}, \ldots, f_{t_{m}-1}$ is a basis of $S_{m}(\Gamma)$. For any non-zero $f \in S_{m}(\Gamma)$, this map can be written as follows:

$$
\mathfrak{a}_{z} \mapsto\left(f_{0}(z) / f(z): \cdots: f_{t_{m}-1}(z) / f(z)\right) .
$$

As with any holomorphic map, this one has attached divisor $\mathfrak{d}$. The divisor $\mathfrak{d}$ is defined by

$$
-\mathfrak{d}=\sum_{\mathfrak{a} \in \mathfrak{R}_{\Gamma}} \min \left(\operatorname{div}\left(\frac{f_{i}}{f}\right)(\mathfrak{a}), 0 \leq i \leq t_{m}-1\right) \mathfrak{a} .
$$


When $t_{m} \geq g+1$, it is very easy to compute $\mathfrak{d}$. By Lemma $2-2$ (vi), we have the following:

$$
\begin{aligned}
-\mathfrak{d} & =\sum_{\mathfrak{a} \in \mathfrak{R}_{\Gamma}} \min \left(\operatorname{div}\left(\frac{f_{i}}{f}\right)(\mathfrak{a}), 0 \leq i \leq t_{m}-1\right) \mathfrak{a} \\
& =\sum_{\mathfrak{a} \in \mathfrak{R}_{\Gamma}} \min \left(\mathfrak{c}_{f_{i}}(\mathfrak{a})-\mathfrak{c}_{f}(\mathfrak{a}), 0 \leq i \leq t_{m}-1\right) \mathfrak{a} \\
& =-\mathfrak{c}_{f}+\sum_{\mathfrak{a} \in \mathfrak{R}_{\Gamma}} \min \left(\mathfrak{c}_{f_{i}}(\mathfrak{a}), 0 \leq i \leq t_{m}-1\right) \mathfrak{a} \\
& =-\mathfrak{c}_{f},
\end{aligned}
$$

where in the last equality we use Lemma 2-9 (and the assumption $t_{m} \geq g+1$ ).

Now, we prove the main result of this section. By Lemma 2-2 (vi), for non-zero $f, g \in$ $S_{m}(\Gamma)$, we have that

$$
\operatorname{div}\left(\frac{f}{g}\right)=\operatorname{div}(f)-\operatorname{div}(g)=\mathfrak{c}_{f}-\mathfrak{c}_{g} .
$$

Thus, the divisors $\mathfrak{c}_{f}$ and $\mathfrak{c}_{g}$ are equivalent. Hence, the corresponding complete linear systems are equal

$$
\left|\mathfrak{c}_{f}\right|=\left|\mathfrak{c}_{g}\right|
$$

Theorem 3-3. Let $m \geq 3$ such that $t_{m} \geq g+1,2$. Then, the holomorphic map (3-1) is attached to the (base point free) complete linear system $\left|\mathfrak{c}_{f}\right|$ where $f \in S_{m}(\Gamma)$ is any non-zero modular form. Moreover, if $t_{m} \geq g+2$, then the divisor $\mathfrak{c}_{f}$ is very ample; in particular, the holomorphic map (3-1) is an embedding.

Proof. By above computation, the divisor attached to the map (3-2) is $\mathfrak{c}_{f}$. The linear system of the holomorphic map (3-2) is by definition equal to

$$
\left\{\operatorname{div}\left(\sum_{i=0}^{t_{m}-1} a_{i} f_{i} / f\right)+\mathfrak{c}_{f} ; \quad a_{0}, \ldots, a_{t_{m}-1} \in \mathbb{C}\right\} .
$$

By Proposition 2-10, it is equal to the complete linear system $\left|\mathfrak{c}_{f}\right|$. This proves the first claim.

By definition $\left|\mathfrak{c}_{f}\right|$ is very ample if it is base-point-free and it defines an embedding. By Proposition 2-10, the complete linear system $\left|\mathfrak{c}_{f}\right|$ is base-point-free for $t_{m} \geq g+1$. By Lemma 2-2 (vi), we obtain we obtain

$$
\operatorname{deg}\left(\mathfrak{c}_{f}\right)=g-1+t_{m} \geq(g-1)+(g+2)=2 g+1 .
$$

By the standard theory of algebraic curves, any divisor of degree $\geq 2 g+1$ is very ample. In fact, we did not have to check that $\left|\mathfrak{c}_{f}\right|$ is base-point free in advance since this holds for any any divisor of degree $\geq 2 g+1$. 
Corollary 3-4. Assume that $m \geq 3$ is given such that $t_{m} \geq g+2$. Then, if $f_{0}, \ldots, f_{t_{m}-1}$ is a basis of $S_{m}(\Gamma)$, then we denote by

$$
\mathcal{C}=\mathcal{C}\left(f_{0}, \ldots, f_{t_{m}-1}\right)
$$

the image of the map (3-1). Then, $\mathcal{C}$ is a irreducible smooth projective curve in $\mathbb{P}^{t_{m}-1}$ which has a degree $t_{m}+g-1$.

Proof. The first claim follows by Chow's theorem. By the standard theory, the degree is given by

by Lemma 2-2 (vi).

$$
\operatorname{deg}(\mathcal{C})=\operatorname{deg}\left(\mathfrak{c}_{f}\right)=t_{m}+g-1,
$$

Corollary 3-6. Assume that $m \geq 3$ is given such that $t_{m} \geq g+2$. Then, for the curve $\mathcal{C}$ given by (3-5), the hyperplane intersection divisor for $x_{0}=0$ is $\mathfrak{c}_{f_{0}}$.

Proof. Indeed, the corresponding map we can write has follows:

$$
\mathfrak{a}_{z} \longmapsto\left(f_{0}(z): \cdots: f_{t_{m}-1}(z)\right)=\left(1: f_{1}(z) / f_{0}(z) \cdots: f_{t_{m}-1}(z) / f_{0}(z)\right) .
$$

Next, by Lemma 2-2 (vi), we note that

$$
\operatorname{div}\left(f_{i} / f_{0}\right)=\operatorname{div}\left(f_{i}\right)-\operatorname{div}\left(f_{0}\right)=\mathfrak{c}_{f_{i}}-\mathfrak{c}_{f_{0}},
$$

where $\mathfrak{c}_{f_{i}}, \mathfrak{c}_{f_{0}} \geq 0$. Finally, Lemma 2-9, shows that if $\mathfrak{c}_{f_{0}}\left(\mathfrak{a}_{\zeta}\right)>0$ for some $\mathfrak{a}_{\zeta} \in \mathfrak{R}_{\Gamma}$, then there exists $i \geq 1$ such that $\mathfrak{c}_{f_{i}}\left(\mathfrak{a}_{\zeta}\right)=0$. Now, the proof is immediate.

Since $f_{0}$ could be any non-zero modular form in $S_{m}(\Gamma)$, we see that the divisors $\mathfrak{c}_{f}$ are just the hyperplane intersection divisors. Hence, their geometric interpretation.

Corollary 3-7. Assume that $m \geq 3$ is given such that $t_{m} \geq g+2$. Then, the field of rational functions $\mathbb{C}\left(\mathfrak{R}_{\Gamma}\right)$ is generated over $\mathbb{C}$ by the rational functions $f_{i} / f_{0}, 1 \leq i \leq t_{m}-1$.

Proof. We denote the homogeneous coordinates on $\mathbb{P}^{t_{m}-1}$ by $\left(x_{0}: \cdots: x_{t_{m}-1}\right)$. We remark that $\mathcal{C}$ does not lie in any hyperplane, and, in particular, not in $x_{0}=0$. Thus, the field of rational functions on $\mathcal{C}$ is generated by $x_{i} / x_{0}, 1 \leq i \leq t_{m}-1$. Now, apply the map (3-1).

\section{Wronskians AND EXPLICIT VERSION OF THEOREM 3-3}

Using, some further results from [8], we can make Theorem 3-3 more explicit. This is the goal of the present section. We start with the following lemma:

Lemma 4-1. Let $m \geq 1$. Then, for any sequence $f_{1}, \ldots, f_{k} \in S_{m}(\Gamma)$, the Wronskian

$$
W\left(f_{1}, \ldots, f_{k}\right)(z) \stackrel{\text { def }}{=}\left|\begin{array}{ccc}
f_{1}(z) & \ldots & f_{k}(z) \\
\frac{d f_{1}(z)}{d z} & \ldots & \frac{d f_{k}(z)}{d z} \\
\frac{d^{k-1} f_{1}(z)}{d z^{k-1}} & \ldots & \frac{d^{k-1} f_{k}(z)}{d z^{k-1}}
\end{array}\right|
$$

is a cuspidal modular form in $S_{k(m+k-1)}(\Gamma)$. 
Proof. This is a standard fact (see [15], ([1], page 162), ([8], Theorem 3-8 (iii)). We leave details to the reader.

We recall the following construction of elements of $S_{m}(\Gamma)$ (see [8], Proposition 2.1). We assume that $m \geq 3$. Put $\epsilon_{\Gamma}=\#(\{ \pm 1\} \cap \Gamma)$. Let $\xi \in X$. Then, $k \geq 0$, the series

$$
\Delta_{k, m, \xi}(z) \stackrel{\text { def }}{=} \frac{(m-1) m \cdots(m+k-1)(2 \sqrt{-1})^{m}}{4 \epsilon_{\Gamma} \pi} \sum_{\gamma \in \Gamma}(\gamma \cdot z-\bar{\xi})^{-k-m} \mu(\gamma, z)^{-m}
$$

converges absolutely and uniformly on compact sets to an element of $S_{m}(\Gamma)$ which satisfies

$$
\left\langle f, \Delta_{k, m, \xi}\right\rangle=\left.\frac{d^{k} f(z)}{d z^{k}}\right|_{z=\xi}, \quad f \in S_{m}(\Gamma), k \geq 0,
$$

where the Petersson inner product on $S_{m}(\Gamma)$ is defined by

$$
\left\langle f_{1}, f_{2}\right\rangle=\int_{\Gamma \backslash X} y^{m} f_{1}(z) \overline{f_{2}(z)} \frac{d x d y}{y^{2}} .
$$

We put

$$
W_{k}(z, \xi) \stackrel{\text { def }}{=}\left|\begin{array}{ccc}
\Delta_{0, m, \xi}(z) & \cdots & \Delta_{k, m, \xi}(z) \\
\frac{d \Delta_{0, m, \xi}(z)}{d z} & \cdots & \frac{d \Delta_{k, m, \xi}(z)}{d z} \\
\frac{d^{k} \Delta_{0, m, \xi}(z)}{d z^{k}} & \cdots & \frac{d^{k} \Delta_{k, m, \xi}(z)}{d z^{k}}
\end{array}\right|
$$

The next lemma collects some properties of those functions.

Lemma 4-5. Let $m \geq 3$ and $k \geq 0$. Let $\xi \in X$. Then, we have the following:

(i) $W_{k}(\cdot, \xi) \in S_{(k+1)(m+k)}(\Gamma)$.

(ii) The function $W_{k}(\xi) \stackrel{\text { def }}{=} W_{k}(\xi, \xi)$ is real-analytic and non-negative function on $X$.

(iii) We have $W_{k}(\gamma . \xi)=|\mu(\gamma, \xi)|^{2(k+1) m+2 k(k+1)} W_{k}(\xi)$, for all $\gamma \in \Gamma$ and $\xi \in X$.

(iv) The sequence $\Delta_{0, m, \xi}, \ldots, \Delta_{k, m, \xi}$ is linearly independent if and only if $W_{k}(\xi)=0$.

(v) If $k \geq t_{m}$, then $W_{k}$ is identically zero.

(vi) The set of zeroes of $W_{k}$ is $\Gamma$-invariant. If $W_{k}$ is not identically zero, then the zeroes of $W_{k}$ belongs to finitely many $\Gamma$-orbits.

(vii) For any non-elliptic point $\xi$ which satisfies $W_{t_{m}-1}(\xi) \neq 0$, we have $k_{\xi, m}=t_{m}-1$.

(viii) Let $\xi$ be an elliptic point. Then $W_{k}(\xi)=0$ for all $k \geq 0$ if the order of $\Gamma_{\xi}$ does not divide $m$.

(ix) Let $\xi$ be an elliptic point. Then $W_{k}(\xi)=0$ for all $k \geq 1$. 
Proof. (i) follows from Lemma 4-1. Next, using (4-3), we can write (4-4) in the following form:

$$
W_{k}(z, \xi)=\left|\begin{array}{lll}
\left\langle\Delta_{0, m, \xi}, \Delta_{0, m, z}\right\rangle & \cdots & \left\langle\Delta_{k, m, \xi}, \Delta_{0, m, z}\right\rangle \\
\left\langle\Delta_{0, m, \xi}, \Delta_{1, m, z}\right\rangle & \cdots & \left\langle\Delta_{k, m, \xi}, \Delta_{1, m, z}\right\rangle \\
& \cdots & \\
\left\langle\Delta_{0, m, \xi}, \Delta_{k, m, z}\right\rangle & \cdots & \left\langle\Delta_{k, m, \xi}, \Delta_{k, m, z}\right\rangle
\end{array}\right| .
$$

This shows that the Gramm determinant of the sequence of modular forms $\Delta_{0, m, \xi}, \ldots \Delta_{k, m, \xi}$ is $W_{k}(\xi)$. This proves (iv). Also, one of the basic property of the Gramm determinant implies $W_{k}(\xi) \geq 0$ for all $\xi \in X$. This proves that the function in (ii) is non-negative. It is real analytic by ([8], Proposition 2-7 (i)). This completes the proof of (iii). Next, (iv) is a consequence of Lemma 4-1 and the following obvious consequence of (4-6) $W_{k}(z, \xi)=$

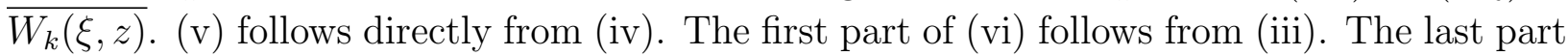
of (vi) follows from the following observation. By (iv), the claim is sufficient to establish for $k=t_{m}-1$. But, by the proof of ([8], Theorem 3-5 (iii)), those orbits are determined as the zeroes of the cuspidal modular form given by the Wronskian $W\left(f_{1}, \ldots, f_{t_{m}}\right)$, attached to a basis $f_{1}, \ldots, f_{t_{m}}$ of $S_{m}(\Gamma)$. Finally, (vii) follows from (vi) and ([8], Theorem 3-5 (iii)). We prove (viii). We observe that $\gamma \longrightarrow \mu(\gamma, \xi)$ is a character $\Gamma_{\xi} \longrightarrow \mathbb{C}^{\times}$by the cocycle relation

$$
\mu\left(\gamma_{1} \gamma_{2}, \xi\right)=\mu\left(\gamma_{1}, \gamma_{2} . \xi\right) \mu\left(\gamma_{2}, \xi\right)=\mu\left(\gamma_{1}, \xi\right) \mu\left(\gamma_{2}, \xi\right) .
$$

Its kernel is trivial. Indeed, if

$$
\gamma=\left(\begin{array}{ll}
a_{\gamma} & b_{\gamma} \\
c_{\gamma} & d_{\gamma}
\end{array}\right) \in \Gamma_{\xi}
$$

satisfies

$$
c_{\gamma} \xi+d_{\gamma}=\mu(\gamma, \xi)=1
$$

then taking the imaginary part we find $c_{\gamma}=0$ and $d_{\gamma}=1$. Also

$$
a_{\gamma} \xi+b_{\gamma}=\frac{a_{\gamma} \xi+b_{\gamma}}{c_{\gamma} \xi+d_{\gamma}}=\gamma \cdot \xi=\xi
$$

implies $a_{\gamma}=1$ and $b_{\gamma}=0$. Hence, the group $\Gamma_{\xi}$ can be considered as a subgroup of $\mathbb{C}^{\times}$. In particular, it is cyclic. So, if the order of $\Gamma_{\xi}$ does not divide $m$, we can find $\gamma \in \Gamma_{\xi}$ such that $\mu(\gamma, \xi)^{m} \neq 1$. Hence, for $f \in S_{m}(\Gamma), f(\xi)=f(\gamma . \xi)=\mu(\gamma, \xi)^{m} f(\xi)$ implies $f(\xi)=0$. In particular,

$$
\left\langle\Delta_{0, m, \xi}, \Delta_{0, m, \xi}\right\rangle=\Delta_{0, m, \xi}(\xi)=0 .
$$

Hence, $\Delta_{0, m, \xi}=0$. Since $W_{k}(\xi)$ can be computed using (4-6) with $z=\xi$, the first row there consists of 0 's in our case. Thus, we have proved that if $m$ is not divisible by the order of $\Gamma_{\xi}$, then $W_{k}(\xi)=0$ for all $k \geq 0$. This proves (viii). Finally, we prove (ix). By (viii) we may assume that the order of $\Gamma_{\xi}$ divides $m$. Then, since the order of $\Gamma_{\xi}$ is greater than 2 (this fact is elementary ${ }^{2}$ ), then $W_{1}(\xi)=0$ by (i). Now, (iv) implies that $\Delta_{0, m, \xi}$ and $\Delta_{1, m, \xi}$ are linearly dependent. Thus, the sequence $\Delta_{0, m, \xi}, \ldots, \Delta_{k, m, \xi}$ is linearly dependent for $k \geq 1$. Hence, $W_{k}(\xi)=0$ by (iv).

\footnotetext{
${ }^{2}$ If not, then by the definition of an elliptic point (see the begining of Section 2), we find that the order of $\Gamma_{\xi}$ is equal to 2. But in $S L_{2}(\mathbb{R})$ elements of order $\leq 2$ are \pm 1 . Then, we would get $e_{\xi}=1$ which is a contradiction with the definition of an elliptic point.
} 
Let $\xi \in X$. Then, we define for all $k \geq 0$

$$
\begin{aligned}
& \Xi_{k, m, \xi}(z)=\left|\begin{array}{cccc}
\left\langle\Delta_{0, m, \xi}, \Delta_{0, m, \xi}\right\rangle & \cdots & \left\langle\Delta_{0, m, \xi}, \Delta_{k-1, m, \xi}\right\rangle & \Delta_{0, m, \xi} \\
\left\langle\Delta_{1, m, \xi}, \Delta_{0, m, \xi}\right\rangle & \cdots & \left\langle\Delta_{1, m, \xi}, \Delta_{k-1, m, \xi}\right\rangle & \Delta_{1, m, \xi} \\
& \cdots & \cdots & \cdots \\
\left\langle\Delta_{k, m, \xi}, \Delta_{0, m, \xi}\right\rangle & \cdots & \left\langle\Delta_{k, m, \xi}, \Delta_{k-1, m, \xi}\right\rangle & \Delta_{k, m, \xi}
\end{array}\right| \\
& =\left|\begin{array}{cccc}
\Delta_{0, m, \xi}(\xi) & \cdots & \left.\frac{d^{k-1} \Delta_{0, m, \xi}}{d_{1, m, \xi}(\xi)}\right|_{z=\xi} & \Delta_{0, m, \xi} \\
\Delta_{1,1}-1 & \left.\frac{d^{k-1} \Delta_{1, m, \xi}}{d z^{k-1}}\right|_{z=\xi} & \Delta_{1, m, \xi} \\
\Delta_{k, m, \xi}(\xi) & \cdots & \left.\frac{d^{k-1} \Delta_{k, m, \xi}}{d z^{k-1}}\right|_{z=\xi} & \Delta_{k, m, \xi}
\end{array}\right| .
\end{aligned}
$$

We develop $\Xi_{k, m, \xi}$ into power series centered at $\xi$ :

$$
\Xi_{k, m, \xi}(z)=\sum_{j=k}^{\infty} W_{k, j}(\xi)(z-\xi)^{j}
$$

where we let

Note that

$$
W_{k, j}(\xi)=\left|\begin{array}{cccc}
\Delta_{0, m, \xi}(\xi) & \ldots & \left.\frac{d^{k-1} \Delta_{0, m, \xi}}{d z^{k-1}}\right|_{z=\xi} & \left.\frac{d^{j} \Delta_{0, m, \xi}}{d z^{j}}\right|_{z=\xi} \\
\Delta_{1, m, \xi}(\xi) & \ldots & \left.\frac{d^{k-1} \Delta_{1, m, \xi}}{d z^{k-1}}\right|_{z=\xi} & \left.\frac{d^{j} \Delta_{1, m, \xi}}{d z^{j}}\right|_{z=\xi} \\
& \cdots & \ldots & \ldots \\
\Delta_{k, m, \xi}(\xi) & \ldots & \left.\frac{d^{k-1} \Delta_{k, m, \xi}}{d z^{k-1}}\right|_{z=\xi} & \left.\frac{d^{j} \Delta_{k, m, \xi}}{d z^{j}}\right|_{z=\xi}
\end{array}\right| .
$$

$$
W_{k, k}(\xi)=W_{k}(\xi)
$$

Thus, $\nu_{z-\xi}\left(\Xi_{k, m, \xi}\right)=k$ if and only if $W_{k}(\xi) \neq 0$.

Lemma 4-9. Let $m \geq 3$ such that $S_{m}(\Gamma) \neq 0$. Assume that $\xi \in X$ satisfies $W_{t_{m}-1}(\xi) \neq 0$. Then, we have the following:

(i) The modular forms $\Xi_{0, m, \xi}, \Xi_{1, m, \xi}, \ldots, \Xi_{t_{m}-1, m, \xi}$ form the basis of $S_{m}(\Gamma)$.

(ii) We have $\nu_{z-\xi}\left(\Xi_{k, m, \xi}\right)=k$ for $0 \leq k \leq t_{m}-1$.

(iii) We may take $f_{\xi, m}=\Xi_{t_{m}-1, m, \xi}$, up to a scalar $\neq 0$. In particular, $k_{\xi, m}=t_{m}-1$.

Proof. (i) is obvious from (4-7) and (4-8) combined with Lemma 4-5 (iv). (ii) follows from the comments before the statement of the lemma combined with the fact that $W_{t_{m}-1}(\xi) \neq 0$ implies $W_{k}(\xi) \neq 0$ for all $0 \leq k \leq t_{m}-1$. (See Lemma 4-5 (iv).) Finally, (iii) follows from Lemma 4-5 (vii).

Now, we prove the main result of the present section. The present theorem is a slight generalization of Theorem 3-3 since we are dealing with specific linear systems.

Theorem 4-10. Let $m \geq 3$ such that $t_{m} \geq g+2$. Then, for $1 \leq k \leq t_{m}-1$, we define a continuous map $\left\{W_{t_{m}-1}(\xi) \neq 0\right\} \times \mathfrak{R}_{\Gamma} \longrightarrow \mathbb{P}^{k}$ by $\left(\xi, \mathfrak{a}_{z}\right) \mapsto\left(\Xi_{t_{m}-1-k, m, \xi}(z): \cdots: \Xi_{t_{m}-1, m, \xi}(z)\right)$ which is real analytic in $\xi$ and holomorphic in $z$. Moreover, for each $\xi$ it sends $\mathfrak{a}_{\xi}$ to $(1: 0: \cdots: 0)$. Finally, if $k \geq g+1$ (which is satisfied for $k=t_{m}-1$ ), then the map 
is attached to base point free complete linear system $\left|k \mathfrak{a}_{\xi}+\mathfrak{d}_{\xi, m}\right| ; k \mathfrak{a}_{\xi}+\mathfrak{d}_{\xi, m}$ is very ample and it defines an embedding of $\mathfrak{R}_{\Gamma}$ into $\mathbb{P}^{k}$. The field of rational functions on $\mathfrak{R}_{\Gamma}$ is generated over $\mathbb{C}$ by

$$
\frac{\Xi_{t_{m}-g-2, m, \xi}}{\Xi_{t_{m}-1, m, \xi}}, \ldots, \frac{\Xi_{t_{m}-2, m, \xi}}{\Xi_{t_{m}-1, m, \xi}} .
$$

Proof. The map is well-defined by Lemma 4-9 (i). The fact that the map is continuous, real analytic in $\xi$, and holomorphic in $z$ follows easily from ([8], Proposition 2-7) and its definition. It maps $\mathfrak{a}_{\xi}$ to $(1: 0: \cdots: 0)$ by Lemma $4-9$ (ii).

Next, the assumption $t_{m} \geq g+2$ implies that $t_{m}-1 \geq 1$. Hence, Lemma 4-5 (ix) imply that $\xi$ is not elliptic when $W_{t_{m}-1}(\xi) \neq 0$. Then, Lemma 4-9 (iii) implies that $k_{\xi}=t_{m}-1$. Hence, (2-5) implies that the degree of $k \mathfrak{a}_{\xi}+\mathfrak{d}_{\xi, m}$ is $k+g$. Assuming that $k \geq g+1$ we obtain the divisor $k \mathfrak{a}_{\xi}+\mathfrak{d}_{\xi, m}$ of degree $k+g \geq 2 g+1$. By the standard theory, this implies that divisor $k \mathfrak{a}_{\xi}+\mathfrak{d}_{\xi, m}$ very ample. In particular, $\left|k \mathfrak{a}_{\xi}+\mathfrak{d}_{\xi, m}\right|$ is base-point-free.

By Lemma 4-9 (ii), (iii), Lemma 2-7, and Lemma 2-8, the basis of $L\left(k \mathfrak{a}_{\xi}+\mathfrak{d}_{\xi, m}\right)$ is given by

$$
\frac{\Xi_{t_{m}-1-k, m, \xi}}{\Xi_{t_{m}-1, m, \xi}}, \ldots, \frac{\Xi_{t_{m}-1, m, \xi}}{\Xi_{t_{m}-1, m, \xi}}
$$

Thus, the map attached to the base point free complete linear system $\left|k \mathfrak{a}_{\xi}+\mathfrak{d}_{\xi, m}\right|$ is exactly the map $\mathfrak{R}_{\Gamma} \longrightarrow \mathbb{P}^{k}$ by $\mathfrak{a}_{z} \mapsto\left(\Xi_{t_{m}-1-k, m, \xi}(z): \cdots: \Xi_{t_{m}-1, m, \xi}(z)\right)$ which is embedding since the divisor $k \mathfrak{a}_{\xi}+\mathfrak{d}_{\xi, m}$ very ample.

The very last claim has the same proof as Corollary 3-7.

We remark that for $k=g+1$, we constructed a family of explicit embeddings of $\mathfrak{R}_{\Gamma}$ into $\mathbb{P}^{g+1}$. They are quite different than usual embeddings into $\mathbb{P}^{g+1}$ constructed with the aid of the complete linear system $(2 g+1) \mathfrak{a}$, where $\mathfrak{a} \in \mathfrak{R}_{\Gamma}$ is any point. For $m$ large enough, we have that $t_{m} \geq 2 g+2$. Then, among the maps in Theorem $4-10$ is the map attached to $\left|(2 g+1) \mathfrak{a}_{\xi}+\mathfrak{d}_{\xi, m}\right|$. It defines an embedding into $\mathbb{P}^{2 g+1}$. Choosing appropriately the $(g+1)-$ dimensional linear subspace $E$ of $\mathbb{P}^{2 g+1}$, we may project the curve to get the curve in $E$ which is in fact the one that comes from $\left|(2 g+1) \mathfrak{a}_{\xi}\right|$. One may write down the equations for this in terms of the divisor $\mathfrak{d}_{\xi, m}$.

\section{ON THE DIVISOR $\mathfrak{d}_{\xi, m}$}

In this section we consider the case when $\Gamma$ has no cusps and elliptic points. This forces $g \geq 2$. By Lemma $2-2(\mathrm{v}), t_{m}=\operatorname{dim} S_{m}(\Gamma)=(m-1)(g-1) \neq 0$ for $m \geq 3$. Then, we may observe that Lemma 4-9 (iii) shows that $f_{\xi, m}$ can be taken to depend continuously on $\xi$ on the set defined by $W_{t_{m}-1}(\xi) \neq 0$. More precisely, we may let $f_{\xi, m}=\Xi_{t_{m}-1, m, \xi}$. Hence, we may view $(\xi, z) \mapsto f_{\xi, m}(z)$ as a continuous function which is real analytic in $\xi$ and holomorphic in $z$. This will help us understand the divisor $\mathfrak{d}_{\xi, m}$. So far, we only know that it does not contain $\mathfrak{a}_{\xi}$ in its support, it is non-negative, integral, and it has a degree $g$ (see (2-5)). 
Proposition 5-1. Let $W_{t_{m-1}}\left(\xi_{0}\right) \neq 0$. Then, there exists $\delta>0$ and $C^{\infty}$-functions $b_{0}, \ldots, b_{g-1}$ on the set $W_{t_{m}-1}(\xi) \neq 0,\left|\xi-\xi_{0}\right|<\delta$, such that if $r_{1}(\xi), \ldots, r_{g}(\xi)$ are all zeroes of the polynomial

$$
z^{g}+b_{g-1}(\xi) z^{g-1}+\cdots+b_{0}(\xi)=0
$$

then

$$
\mathfrak{d}_{\xi, m}=\sum_{i=1}^{g} \mathfrak{a}_{r_{i}(\xi)}
$$

Proof. We imitate the proof of the Weierstrass preparation theorem. Let $\zeta_{1}, \ldots, \zeta_{l} \in X$ be fixed representatives of points from the support of $\mathfrak{d}_{\xi_{0}, m}$. We select open circles $D_{i}$ with centers $\zeta_{i}, 1 \leq i \leq l$ such that $\gamma \cdot D_{i} \cap D_{j} \neq \emptyset, \gamma \in \Gamma$, implies $\gamma= \pm 1$. In particular, $f_{\xi_{0}, m}\left(\zeta_{i}\right)=0$, but $f_{\xi_{0}, m}(z) \neq 0$ for $z \in D_{i}-\left\{\zeta_{i}\right\}$. We select a circle $C_{i}$ with center $\zeta_{i}$, say $\left|z-\zeta_{i}\right|=r_{i}$ which is completely inside $D_{i}$. Then, for $z \in C_{i}$, we have $f_{\xi_{0}, m}(z) \neq 0$. Thus, there exists $\delta=\delta_{z}>0$ such that $\left|\xi-\xi_{0}\right|<\delta_{z}$ and $|w-z|<\delta_{z}, w \in C_{i}$, implies $f_{\xi}(w) \neq 0$. Because of the compactness of $C_{i}$, there exists $\delta>0$ such that $\left|\xi-\xi_{0}\right|<\delta$ implies $f_{\xi, m}(z) \neq 0$ for all $z \in C_{i}$. Now, we apply the residue theorem to count zeroes of $f_{\xi, m}$ inside each $D_{i}$. We have the following:

$$
\sum_{\substack{\left|\zeta-\zeta_{i}\right|<r_{i} \\ f_{\xi, m}(\zeta)=0}} \nu_{z-\zeta}\left(f_{\xi, m}\right)=\frac{1}{2 \pi \sqrt{-1}} \int_{C_{i}} \frac{\partial f_{\xi, m}(z) / \partial z}{f_{\xi, m}(z)} d z, \text { for }\left|\xi-\xi_{0}\right|<\delta, 1 \leq i \leq l .
$$

Obviously, we have that the left-hand side is continuous in $\xi$. Thus, we have

$$
\sum_{\substack{\left|\zeta-\zeta_{i}\right|<r_{i} \\ f_{\xi, m}(\zeta)=0}} \nu_{z-\zeta}\left(f_{\xi, m}\right)=\nu_{z-\zeta_{i}}\left(f_{\xi_{0}, m}\right), \text { for }\left|\xi-\xi_{0}\right|<\delta, 1 \leq i \leq l
$$

Because of the assumption $\gamma \cdot D_{i} \cap D_{i} \neq \emptyset$ for $\gamma \neq \pm 1$, we see that the support of the divisor

$$
\mathfrak{d}_{\xi, m, i} \stackrel{\text { def }}{=} \sum_{\substack{\left|\zeta-\zeta_{i}\right|<r_{i} \\ f_{\xi, m}(\zeta)=0}} \nu_{z-\zeta}\left(f_{\xi, m}\right) \mathfrak{a}_{\zeta}
$$

consists of $\Gamma$-non-equivalent points $\mathfrak{a}_{\zeta}$ when $\zeta$ ranges over $f_{\xi, m}(\zeta)=0$ in $\left|\zeta-\zeta_{i}\right|<r_{i}$. As it is also $\gamma \cdot D_{i} \cap D_{j} \neq \emptyset$ for $\gamma \neq \pm 1$, we see that for $i \neq j$ the supports of just constructed divisors $\mathfrak{d}_{\xi, m, i}$ and $\mathfrak{d}_{\xi, m, j}$ are disjoint. Finally, because of the same assumptions we have

$$
\mathfrak{d}_{\xi_{0}, m}=\sum_{i=1}^{l} \nu_{z-\zeta_{i}}\left(f_{\xi_{0}, m}\right) \mathfrak{a}_{\zeta_{i}}
$$

In particular,

$$
\sum_{i=1}^{l} \nu_{z-\zeta_{i}}\left(f_{\xi_{0}, m}\right)=\operatorname{deg}\left(\mathfrak{d}_{\xi_{0}, m}\right)=g
$$


By above discussion, we obtain

$$
\operatorname{deg}\left(\sum_{i=1}^{l} \mathfrak{d}_{\xi, m, i}\right)=\sum_{i=1}^{l} \sum_{\substack{\left|\zeta-\zeta_{i}\right|<r_{i} \\ f_{\xi, m}(\zeta)=0}} \nu_{z-\zeta}\left(f_{\xi, m}\right)=\sum_{i=1}^{l} \nu_{z-\zeta_{i}}\left(f_{\xi_{0}, m}\right)=g
$$

Thus, we finally obtain

$$
\mathfrak{d}_{\xi, m}=\sum_{i=1}^{l} \mathfrak{d}_{\xi, m, i}, \quad\left|\xi-\xi_{0}\right|<\delta .
$$

Again, by the residue theorem, for $m \geq 0$, we have the following:

$$
\sum_{\substack{\left|\zeta-\zeta_{i}\right|<r_{i} \\ f_{\xi, m}(\zeta)=0}} \nu_{z-\zeta}\left(f_{\xi, m}\right) \zeta^{m}=\frac{1}{2 \pi \sqrt{-1}} \int_{C_{i}} z^{m} \frac{\partial f_{\xi, m}(z) / \partial z}{f_{\xi, m}(z)} d z, \text { for }\left|\xi-\xi_{0}\right|<\delta, 1 \leq i \leq l .
$$

This implies that the sum

$$
\sum_{i=1}^{l} \sum_{\substack{\left|\zeta-\zeta_{i}\right|<r_{i} \\ f_{\xi, m}(\zeta)=0}} \nu_{z-\zeta}\left(f_{\xi, m}\right) \zeta^{m}
$$

is a $C^{\infty}$-function on $\left|\xi-\xi_{0}\right|<\delta$. In another words, if $r_{1}(\xi), \cdots, r_{g}(\xi)$ are all zeroes of $f_{\xi}$ in the union of the circles $\left|\zeta-\zeta_{i}\right|<r_{i}$ counted with multiplicity, then

$$
r_{1}^{m}(\xi)+\cdots+r_{g}^{m}(\xi), \quad m \geq 0,
$$

is a $C^{\infty}$-function.

Let $\sigma_{1}(\xi), \ldots, \sigma_{g}(\xi)$ be the elementary symmetric polynomials in $r_{1}(\xi), \cdots, r_{g}(\xi)$. These are polynomials in the sums of powers, hence $C^{\infty}$-functions.

\section{REFERENCES}

[1] H. M. FARKas, I. KRA, Theta Constants, Riemann Surfaces and the Modular Group, Graduate Studies in Mathematics 37, American Mathematical Society (2001).

[2] R. Holowinsky, K. Soundararajan, Mass equidistribution for Hecke eigenforms, Ann. of Math. 172 (2010), 1517-1528.

[3] J. Kollár, Shafarevich Maps and Automorphic Forms, Princeton Univ. Press (1995).

[4] T. Miyake, Modular forms, Springer-Verlag (2006).

[5] R. Miranda, Algebraic Curves and Riemann Surfaces, Graduate Studies in Mathematics 5 (1995).

[6] G. Muić, On the Cuspidal Modular Forms for the Fuchsian Groups of the First Kind, Journal of Number Theory 30. No. 1 (2010), 1488-1511.

[7] G. Muić, On the non-vanishing of certain modular forms, International J. of Number Theory Vol. 7, Issue 2 (2011) pp. 351-370.

[8] G. Muić, Modular curves and bases for the spaces of cuspidal modular forms, Ramanujan J. 27 (2012), 181-208.

[9] G. Muić, On the inner product of certain automorphic forms and applications, preprint (http://web.math.pmf.unizg.hr/ gmuic/papers.html).

[10] G. MuIć, On the analytic continuation and non-vanishing of L-functions, preprint (http://web.math.pmf.unizg.hr/ gmuic/papers.html). 
[11] A. Neeman The distribution of Weierstrass points on a compact Riemann surface, Ann. Math. 120 (1984), 317-328.

[12] B. A. Olsen, On Higher Order Weierstrass Points, Ann. Math.95 No. 2 (1972), 357-364.

[13] H. Petersson, Einheitliche Begründung der Vollständigkeitssätze für die Poincaréschen Reihen von reeller Dimension bei beliebigen Grenzkreisgruppen von erster Art, Abh. Math. Sem. Hansischen Univ. 14 (1941), 22-60.

[14] H. Petersson, Über Weierstrasspunkte und die expliziten Darstellungen der automorphen Formen von reeller Dimension, Math. Z. 52 (1949), 32-59.

[15] D. Rohrlich, Weierstrass points and modular forms, Illinois J. Math 29 (1985), 134-141

[16] Z. RudNick, On the asymptotic distribution of zeros of modular forms, Intern. Math. Research Notices No. 34 (2005), 2059-2074.

Department of Mathematics, University of Zagreb, BijeničKa 30, 10000 Zagreb, Croatia E-mail address: gmuic@math.hr 